\title{
Specificity Study on Concentration of Monoamine Transmitters at Acupoint and Effect of Acupuncture on Its Distribution
}

\author{
Ting Luo and Yi Guo \\ College of Acupuncture and Moxibustion, Tianjin University of Traditional Chinese Medicine, Tianjin 300193, China \\ Correspondence should be addressed to Yi Guo; guoyi_168@163.com
}

Received 5 August 2014; Accepted 15 September 2014; Published 2 December 2014

Academic Editor: Zhang Weibo

Copyright (C) 2014 T. Luo and Y. Guo. This is an open access article distributed under the Creative Commons Attribution License, which permits unrestricted use, distribution, and reproduction in any medium, provided the original work is properly cited.

\begin{abstract}
To investigate the distribution of monoamine transmitters at acupoints and effect of acupuncture on it. Take this experiment by means of microdialysis. Twenty rabbits were randomly assigned to two groups (Group A: acupuncture Guanyuan (RN4), Group B: acupuncture nonacupoint which is besides Guanyuan (RN4) $1 \mathrm{~cm}$ ). Before and after acupuncture was taken, tissue fluids both at Zhongwan (RN12) which is on the same meridian as Guanyuan and at a nonacupoint $1 \mathrm{~cm}$ away from Zhongwan were collected through microdialysis, respectively. The collected samples were analyzed to determine concentrations of monoamine transmitters. Epinephrine and 5-HT were detected. An unknown substance was found. Its concentration at acupoint was significantly higher than that at nonacupoint and decreased after acupuncture. Its significant specificity at acupoints suggests that it may play an important role in meridian's activity.
\end{abstract}

\section{Introduction}

Researchers have carried on research from the perspective of biochemistry of the meridians for years. It has proved that ions [1, 2], oxygen metabolism [3], nitric oxide (NO) [4], adenosine [5], and so forth are closely associated with the meridian activities. Some scholars believe that the meridians are the sensitive lines of sympathetic nerve [6]. The transfer process of acupuncture signal depends on a kind of low weight molecular substances moving along the meridian [7]. In addition, it has suggested that acupuncture can stimulate the release of monoamine transmitters in the incision liquid extract from meridian [8]. However, the skin incision as a mechanical stimulation can also cause skin to release certain biochemical substances, and it cannot distinguish whether such substances only release from the meridians. In consideration of this, we carried out the study by means of microdialysis attempted to reduce this interference to a minimum.

\section{Subjects and Methods}

2.1. Experimental Animals. 20 healthy rabbits ( 9 female, 11 male; weighing 2-2.7 kg; provided by the Animal Center of
Tianjin University of Traditional Chinese Medicine) were randomly divided into two groups, each group has 10 rabbits. Group A received manual needle acupuncture at Guanyuan (RN4) acupoint and group B received manual needle acupuncture at nonacupoint besides Guanyuan (RN4) $1 \mathrm{~cm}$.

2.2. Main Instrument. Consider the following: microperistaltic pump (England Watson-Marlow, $205 \mathrm{U}$ ); Shimadzu LC-6A HPLC; CTO-6A column oven; L-ECD-6A electrochemical detector (Sensitivity: $1 \mathrm{AUF})$; C18 column $(10 \mu$, $250 \mathrm{~mm} \times 4.6 \mathrm{~mm}$, Tianjin dimensional chromatographic company).

\subsection{Experimental Methods}

2.3.1. Preparation of the Microdialysis Probe (See Figure 1, Illustration of the Microdiasis Probe (Concentric Type [9])). A 6th stainless steel needle is obliquely inserted into polyethylene plastic pipe, then a capillary plastic tube, whose outside diameter has been stretched in the range of $160-180 \mu \mathrm{m}$, is inserted in the stainless steel needle with $0.3 \mathrm{~cm}$ exposed outside. We advanced the stunt end of steel needle into the polyethylene 


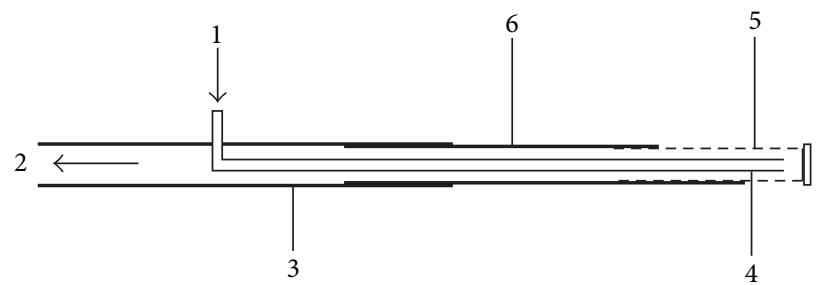

FIGURE 1: Illustration of the microdialysis probe (concentric type). The parts are (1) inlet tubing, (2) outlet tubing, (3) polyethylene plastic pipe, (4) 6th stainless steel needle, (5) dialysis tube, and (6) capillary tube.

TABLE 1: Monoamine neurotransmitter recovery testing.

\begin{tabular}{lccccc}
\hline$N=8$ & NE & E & DHBA & DA & $5-H T$ \\
\hline Recovery \% $(X \pm S D)$ & $6.02 \pm 1.00$ & $7.23 \pm 1.42$ & $68.16 \pm 26.72$ & $5.49 \pm 1.07$ & $2.78 \pm 1.13$ \\
\hline
\end{tabular}

plastic pipe and the tip of the needle exposed for $2 \mathrm{~cm}$. A dialysis tube, molecular weight cut-off is $50 \mathrm{KD}, 1 \mathrm{~cm}$ long, then is carefully advanced from the stainless steel needles tip, and exposed about $2.5 \mathrm{~mm}$ than the needle. Sealed the end of the dialysis tube, the junction between stainless steel needle and dialysis tube, needle and polyethylene plastic pipe, polyethylene plastic pipe, and plastic capillary tube, placed for some time to be solidified.

2.3.2. Probe Detection and Calibrations. In order to test the stability of the probe, a series of experiments have been made. It has been found that single probe, with increasing frequency of use and its recovery, is decremented. And the recovery of several probes has no difference. So the repeated use of single probe is avoided in the experiment.

To further test the stability of the probe recovery, we measured eight-dialysis-probe recovery of monoamine neurotransmitters stability. Methods: eight probes were immersed in a concentration of monoamine neurotransmitters $200 \mathrm{ng} / \mathrm{mL}$ standard solution $100 \mu \mathrm{L}$ (norepinephrine (NE), epinephrine (E), 3,4-dihydroxybenzoic acid (DHBA), dopamine (DA), and 5-hydroxytryptamine (5-HT) join in $0.1 \mathrm{~N} \mathrm{HCl}$ and formulate the standard solution. The standard solution is provided by Sigma), perfused for $30 \mathrm{~min}$, perfusion solution is Ringer's solution, pump speed is $2 \mu \mathrm{L} / \mathrm{min}$, and temperature is $20^{\circ} \mathrm{C}$. And the probe is placed in a stirred homogeneous solution [10]. Collected dialysis solutions are analyzed by HPLC-ECD. For results see Table 1.

2.3.3. Tissue Fluid Collection. Fix the rabbit on rabbit stationary, cut off part of the rabbit hair about $3 \mathrm{~cm}$ in diameter at the monitoring point (Zhongwan (RN12) and $1 \mathrm{~cm}$ besides Zhongwan (RN12)), skin disinfection with iodine-tincture $2 \%$ and alcohol $75 \%$. Two microdialysis probe introducers prepared in advance (75\% alcohol soaked), respectively, were inserted in Zhongwan (RN12) and $1 \mathrm{~cm}$ besides Zhongwan (RN12) with a 45-degree angle, depth of $2 \mathrm{~mm}$. Then two dialysis probes, connected with microperistaltic pump, were carefully inserted into the introducer. Perfusion fluid used Ringer solution having a composition of $\mathrm{Na}^{+} 151.7 \mathrm{mmol} / \mathrm{L}$, $\mathrm{K}^{+} 2.69 \mathrm{mmol} / \mathrm{L}, \mathrm{Ca}^{2+} 1.1 \mathrm{mmol} / \mathrm{L}, \mathrm{Cl}^{-} 152.3 \mathrm{mmol} / \mathrm{L}, \mathrm{pH}: 7.4$.
Perfusion rate is $2 \mu \mathrm{L} / \mathrm{min}$ and was stabilized for $1 \mathrm{~h}$. Collected tissue fluid at two monitor points in a dark place with $0.2 \mathrm{ml}$ centrifuge tubes for $30 \mathrm{~min}$. After this collection, collected another samples also in a dark place with $0.2 \mathrm{ml}$ centrifuge tubes for $30 \mathrm{~min}$. While, at the same time, acupuncture Guanyuan (RN4) and non-acupoint $1 \mathrm{~cm}$ away from Guanyuan in twirling and lifting-thrusting method for $5 \mathrm{~min}$ with 1 cun acupuncture needles, and then retain needles until the collection is finished. Collected samples were put in liquid nitrogen storage tank until being detected.

2.3.4. Detection Method. A total of 80 samples were collected and detected by HPLC-ECD. The chromatographic conditions were as follows: C18 column $(4.6 \mathrm{~mm} \times 250 \mathrm{~mm}$, $10 \mu)$ phosphate buffer ( $\mathrm{pH} 3.2)$ as mobile phase at a flow rate of $0.8 \mathrm{~mL} / \mathrm{min}$. The standard solution is prepared by adding $0.1 \mathrm{~N} \mathrm{HCl}$ in Norepinephrine (NE), epinephrine (E), 3,4-dihydroxybenzoic acid (DHBA), dopamine (DA) and 5hydroxytryptamine $(5-\mathrm{HT})$ which are produced by Sigma company.

2.4. Statistical Analysis. The data were analyzed using SPSS 11.0 software. Testing was performed with Paired-samples $T$ test.

\section{Results}

Epinephrine (E) and 5-HT are detected in a few samples with low concentrations. In addition to E and 5-HT (see Figure 2), most samples have only one peak, and retention time is between 5.8 and $6.1 \mathrm{~min}$. It cannot be concluded what it is. For standards spectra see Figure 2.

Through study on the spectrum of the high peak of the unknown substance, we find that (1) there was a very significant difference $(P<0.01)$ on area of the unknown substance between Zhongwan (RN12) and nonacupoint besides Zhongwan (RN12) $1 \mathrm{~cm}$ (Table 2). (2) The area of the unknown substance reduced after acupuncture Guanyuan (RN4) (Table 3). It has a significant difference $(P<0.05)$. (3) Acupuncture Guanyuan (RN4) also can make area reduced at nonacupoint, but there was no significant difference 


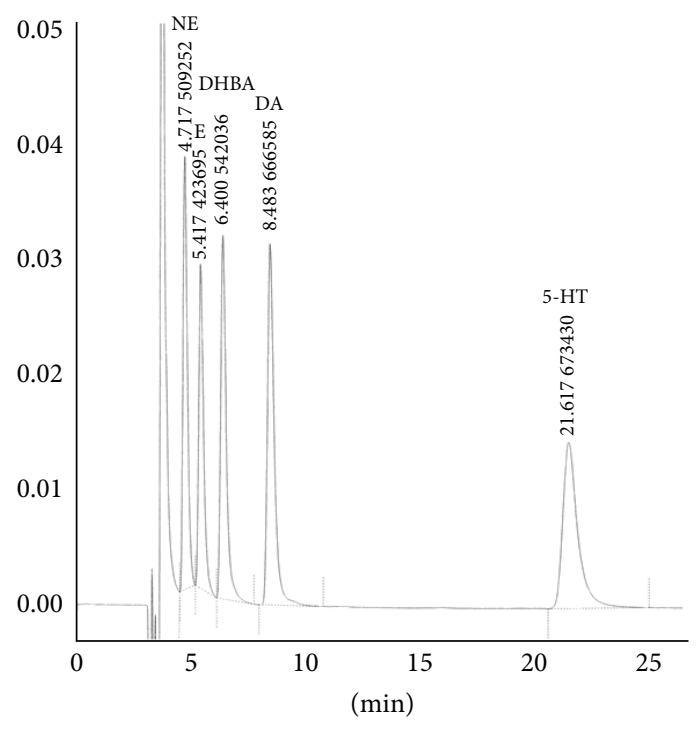

(a)

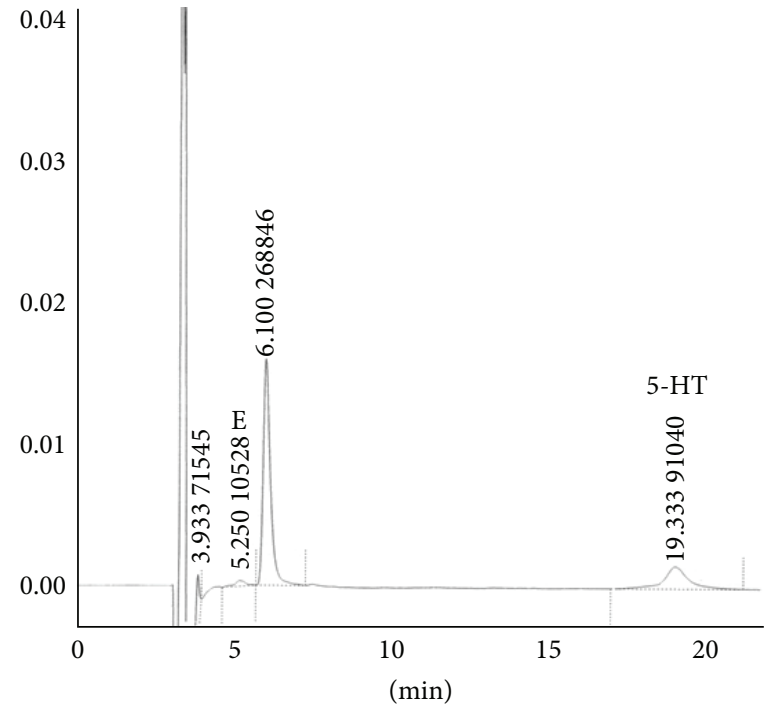

(b)

FIGURE 2: Chromatogram of monoamine transmitter at rabbit's acupoint (a) standard solution (b) dialysate from rabbit's acupoint.

TABLE 2: Comparison of the unknown peak area between acupoint and nonacupoint.

\begin{tabular}{lccc}
\hline & Zhongwan $(\mathrm{RN} 12)(n=20)$ & Nonacupoint $(n=20)$ & Difference \\
\hline Area & $333580.95 \pm 174941.38$ & $204789.60 \pm 91674.55$ & $128791.35 \pm 179245.84^{*}$ \\
\hline${ }^{*} P<0.01$ & & &
\end{tabular}

TABLE 3: Changes of unknown peak area at acupoint after acupuncturing the acupoint.

\begin{tabular}{lccc}
\hline & & Zhongwan (RN12) & \\
& Before acupuncture & After acupuncture & Difference \\
\hline Area & $342006.30 \pm 169110.99$ & $237584.60 \pm 88476.09$ & $104421.70 \pm 129672.31^{*}$ \\
\hline${ }^{*} P<0.05$. & & & \\
\end{tabular}

$(P>0.05)$ (Table 4). (4) Acupuncture nonacupoint makes area increased at the point of Zhongwan (RN12), but there was no significant difference $(P>0.05)$ (Table 5). (5) The area of nonacupoint besides Zhongwan (RN12) $1 \mathrm{~cm}$ increased after acupuncture nonacupoint away from GuanYuan (RN4) $1 \mathrm{~cm}$, and there was no significant difference $(P>0.05)$ (Table 6).

\section{Discussion}

4.1. Experimental Results Analysis. In this study, it has observed that monoamine neurotransmitter levels in dialysate of acupoints and nonacupoints is very low. It is because of two reasons: firstly because the concentration itself is very low at acupoints and nonacupoints and secondly it is due to the microdialysis probes' low recovery especially that of 5 -HT, its recovery is less than $3 \%$ (see Table 1 monoamine neurotransmitters recovery testing).

Nevertheless, E and 5-HT had been tested in the experiment. It is generally believed that E only exists in the outer periphery of the adrenal medulla cells, so some scholars think that this will certainly have meaningful impact on the study of sympathetic substances on the skin [11]. It has detected very low concentrations of 5 -HT at points. It is seen from the detected 27 cases in the sample that there are no differences at 5-HT concentration between acupoint and nonpoint. Regardless of acupoint or nonpoint, concentration of 5-HT in the Guanyuan acupuncturing group is more than the nonacupoint acupuncture group. This trend should be further verified in further experiment by improving recovery of microdialysis probe.

The study also found an unknown substance in the spectrum. It has suggested that (a) the distribution of the unknown substance has significant specificity and the concentration at acupoints is greater than that of nonacupoints, (b) acupuncturing acupoints can make its concentration of acupoints on the same meridian reduced, (c) while acupuncturing acupoints, it has little effect or no effect on its concentration of nonacupoints, (d) it has little effect or no effect on acupoints or nonacupoints, while it is stick nonacupoint. It has also suggested that the unknown substance has a specificity at the meridian and plays a role in the meridian activities. 
TABLE 4: Changes of unknown peak area at nonacupoint after acupuncturing the acupoint.

\begin{tabular}{|c|c|c|c|c|}
\hline & \multicolumn{4}{|c|}{ Away from Zhongwan $(\mathrm{RN} 12) 1 \mathrm{~cm}(n=10)$} \\
\hline & Before acupuncture & After acupuncture & Difference & $T$ \\
\hline Area & $221634.70 \pm 100452.24$ & $204506.10 \pm 197752.34$ & $17128.6 \pm 202874.19^{*}$ & 0.267 \\
\hline
\end{tabular}

TABLE 5: Changes of unknown peak area at acupoint after acupuncturing the nonacupoint.

\begin{tabular}{|c|c|c|c|c|}
\hline & \multicolumn{4}{|c|}{ Zhongwan (RN12) $(n=10)$} \\
\hline & Before acupuncture & After acupuncture & Difference & $T$ \\
\hline Area & $330670.60 \pm 186440.01$ & $360456.50 \pm 242999.06$ & $-29785.9 \pm 313007.28^{*}$ & -0.301 \\
\hline
\end{tabular}

${ }^{*} P>0.05$.

TABLE 6: Changes of unknown peak area at nonacupoint after acupuncturing the nonacupoint.

\begin{tabular}{|c|c|c|c|c|}
\hline & \multicolumn{4}{|c|}{ Away from Zhongwan (RN12) $1 \mathrm{~cm}(n=10)$} \\
\hline & Before acupuncture & After acupuncture & Difference & $T$ \\
\hline Area & $187944.50 \pm 83791.55$ & $200214.30 \pm 153597.45$ & $-12269.8 \pm 199318.27^{*}$ & -0.195 \\
\hline
\end{tabular}

${ }^{*} P>0.05$.

To learn more about the structure of this unknown matter, we used liquid chromatography-mass spectrometry to analyze the dialysis samples. However, because of the high sensitivity of inorganic ions in dialysate solution, it has overshadowed the spectra of the unknown substance. The analysis results are not ideal. And due to the restriction of the sample size, it does not seek an effective method for separating inorganic salts. Therefore, the analysis of unknown structure did not succeed.

In the study of incision fluid along meridian, Liu has detected high concentration of noradrenaline (NA or NE) in some samples, and as for adrenaline (E), he thought it is due to the lack of experimental injection volume it did not detect [11]. Comparing to spectrum of the incision fluid, it is very similar to our experimental spectrum. Liu believes that the peaks that retention time is $7.56 \mathrm{~min}$ and $26.63 \mathrm{~min}$ are mainly intermediate metabolites of catecholamine. It provides some ideas for our future research.

4.2. Function of Monoamine Neurotransmitters In Vivo and Relationship with Meridian Biochemical Studies. Studies have shown that skin of higher animal contains norepinephrine and epinephrine, but no dopamine [12]. Norepinephrine in skin is released primarily by sympathetic nerve endings, while the adrenaline is released by pheochromocytoma scattered in the skin. Experiments show that acupuncture can cause skin to release NA, and the release follows the meridians. NA acts on $\alpha$ receptor, and while $\alpha$ receptor is blocked, it can cause the block of propagated sensation along meridian and acupuncture effect. The agonists of $\alpha$ receptor injected into skin can produce a strong acupuncture effect. Obviously, catecholamines in skin mediate the transduction of meridians and effects of acupuncture. It can infer that the release of NA in meridians by acupuncturing is caused by sympathetic reflex, and neurotransmitters directly diffuse along meridians [13]. Therefore, some scholars believe that the substance of the meridian is sympathetic and sensitive lines in vivo [6]. And by using macroautoradiography method it shows a series of sympathetic substance lines [7]. The skin NE and $3 \mathrm{H}-\mathrm{NE}$ concentration release in acupoints was significantly higher than those in nonacupoints and nonmeridian controls. Consistently, enhanced 3H-NE synthesis/release in acupoints/meridians is facilitated by the presence of an exogenous NO donor and inhibited by an inhibitor of NO synthesis [14]. And studies show that electroacupuncture stimulation induces a significant release of NO and cGMP in the acupuncture acupoint but not in the nonmeridian area [15].5-HT is a kind of pain producing substance in peripheral, and some scholars have found that acupuncture can reduce 5HT and DA levels at inflammation area [16]. In conclusion, monoamine neurotransmitters have a certain relationship with acupuncture in peripheral mechanism of acupuncture.

\section{Conflict of Interests}

The authors declare that there is no conflict of interests regarding the publication of this paper.

\section{Acknowledgment}

The study was supported by the National Natural Science Foundation of China, the project titled regulatory mechanism of the initial kinetic study of acupuncture effects no. 81173204 .

\section{References}

[1] Y. Guo, Y. Zhang, W. Miao et al., "Calcium is one of the key factors in meridian actives," Zhong Guo Zhong Yi Ji Chu Yi Xue Za Zhi, vol. 4, no. 7, pp. 49-51, 1998.

[2] W. Xiuyun, Y. Guo, Z. Yanjun, and X. Tangping, "Acupuncture on acupoint's $\mathrm{K}^{+}$concentrations in rabbits," Zhong Guo Zhong Yi Ji Chu Yi Xue Za Zhi, vol. 4, no. 1, pp. 48-50, 1998. 
[3] W. Zhang, H. Li, and R. Xu, "Observation on skin exhaled volume of carbon dioxide on meridian lines under the influence of acupuncture," Zhong Guo Zhen Jiu, no. 1, pp. 39-42, 1996.

[4] Y. Ha, M. Kim, J. Nah, M. Suh, and Y. Lee, "Measurements of location-dependent nitric oxide levels on skin surface in relation to acupuncture point," Evidence-Based Complementary and Alternative Medicine, vol. 2012, Article ID 781460, 7 pages, 2012.

[5] T. Takano, X. Chen, F. Luo et al., "Traditional acupuncture triggers a local increase in adenosine in human subjects," Journal of Pain, vol. 13, no. 12, pp. 1215-1223, 2012.

[6] L. Liu, A. Peng, J. Pan et al., "Sympathetic nervous sensitive lines and meridian essence," Zhong Guo Zhen Jiu, vol. 21, no. 5, pp. 285-289, 2001.

[7] L. Liyuan, H. Zhang, and P. Juan, "Development of sympathetic substance lines on the skin and its relationship with meridian essence," Zhong Guo Zhen Jiu, vol. 23, no. 1, pp. 23-26, 2003.

[8] L. liyuan and F. Jingyu, "Experimental study on acupuncture meridian skin to promote the release of catecholamines," Shanghai Zhen Jiu Za Zhi, vol. 16, no. 4, pp. 32-34, 1997.

[9] C. S. Chaurasia, "In vivo microdialysis sampling: theory and applications," Biomedical Chromatography, vol.13, no. 5, pp. 317332, 1999.

[10] J. A. Stenken, "Methods and issues in microdialysis calibration," Analytica Chimica Acta, vol. 379, no. 3, pp. 337-358, 1999.

[11] L. Liu, Classical and Modern Meridian, Beijing Medical University and China Xie-He Medical University Joint Publishing House, Beijing, China, 1997.

[12] T. V. Kozyreva, E. Y. Tkachenko, V. P. Kozaruk, T. V. Latysheva, and M. A. Gilinsky, "Effects of slow and rapid cooling on catecholamine concentration in arterial plasma and the skin," American Journal of Physiology-Regulatory Integrative and Comparative Physiology, vol. 276, no. 6, pp. R1668-R1672, 1999.

[13] L. Liyuan, "Information pathway of skin and meridians," Zhong Guo Zhong Yi Ji Chu Yi Xue Za Zhi, vol. 5, no. 8, pp. 47-51, 1999.

[14] J. X. Chen, B. O. Ibe, and S. X. Ma, "Nitric oxide modulation of norepinephrine production in acupuncture points," Life Sciences, vol. 79, no. 23, pp. 2157-2164, 2006.

[15] N. T. Jou and S. X. Ma, "Responses of nitric oxide-cGMP release in acupuncture point to electroacupuncture in human skin in vivo using dermal microdialysis," Microcirculation, vol. 16, no. 5, pp. 434-443, 2009.

[16] L. Fanrong, L. Rong, L. Yuxing et al., "Experimental study of the effect of local inflammation 5-HT, NE, DA content relationship after electroacupuncture analgesia," Zhong Guo Zhong Yi Ji Chu Yi Xue Za Zhi, vol. 7, no. 11, pp. 52-55, 2001. 


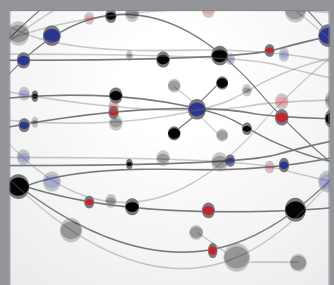

The Scientific World Journal
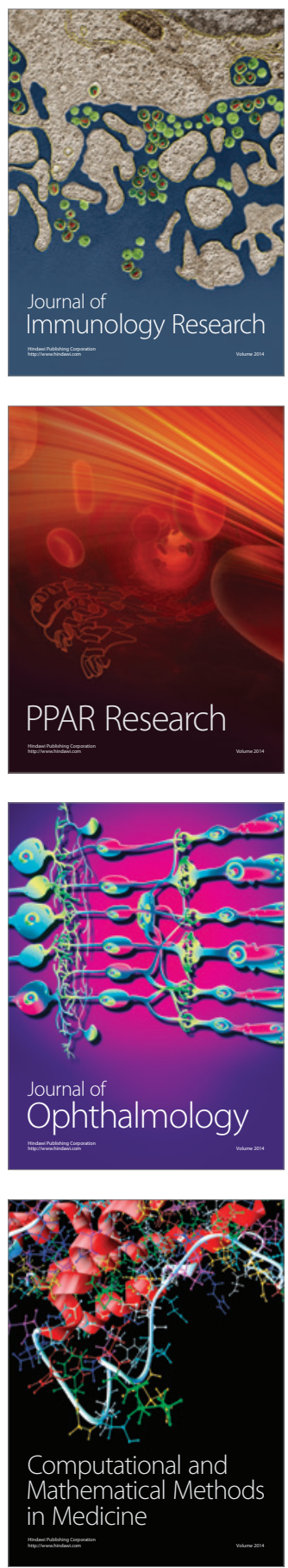

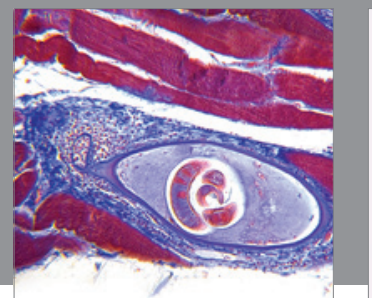

Gastroenterology

Research and Practice
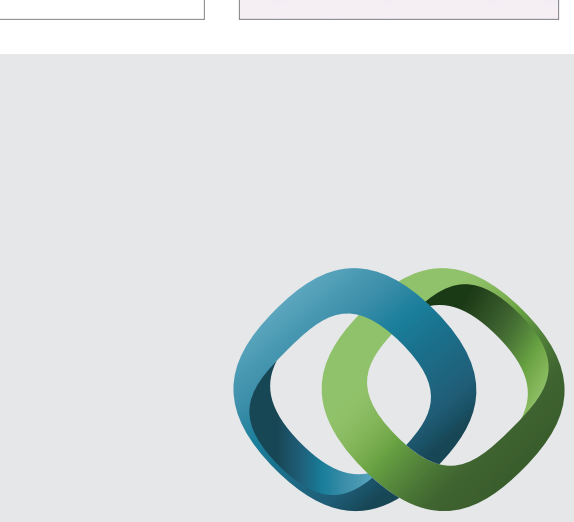

\section{Hindawi}

Submit your manuscripts at

http://www.hindawi.com
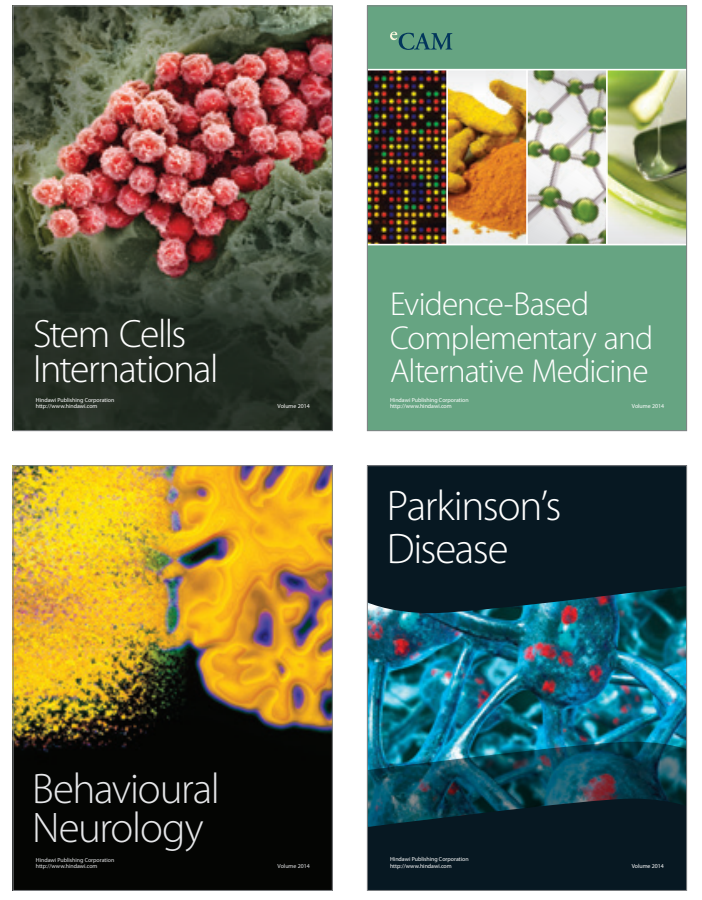
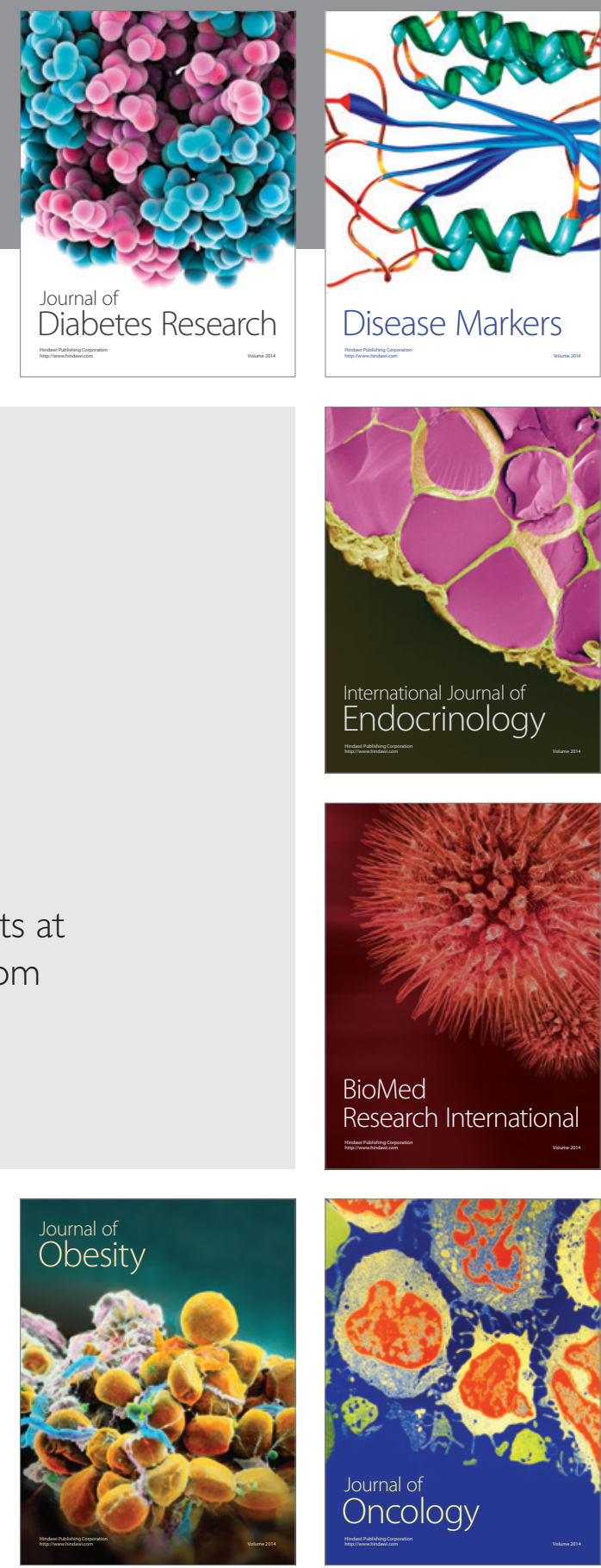

Disease Markers
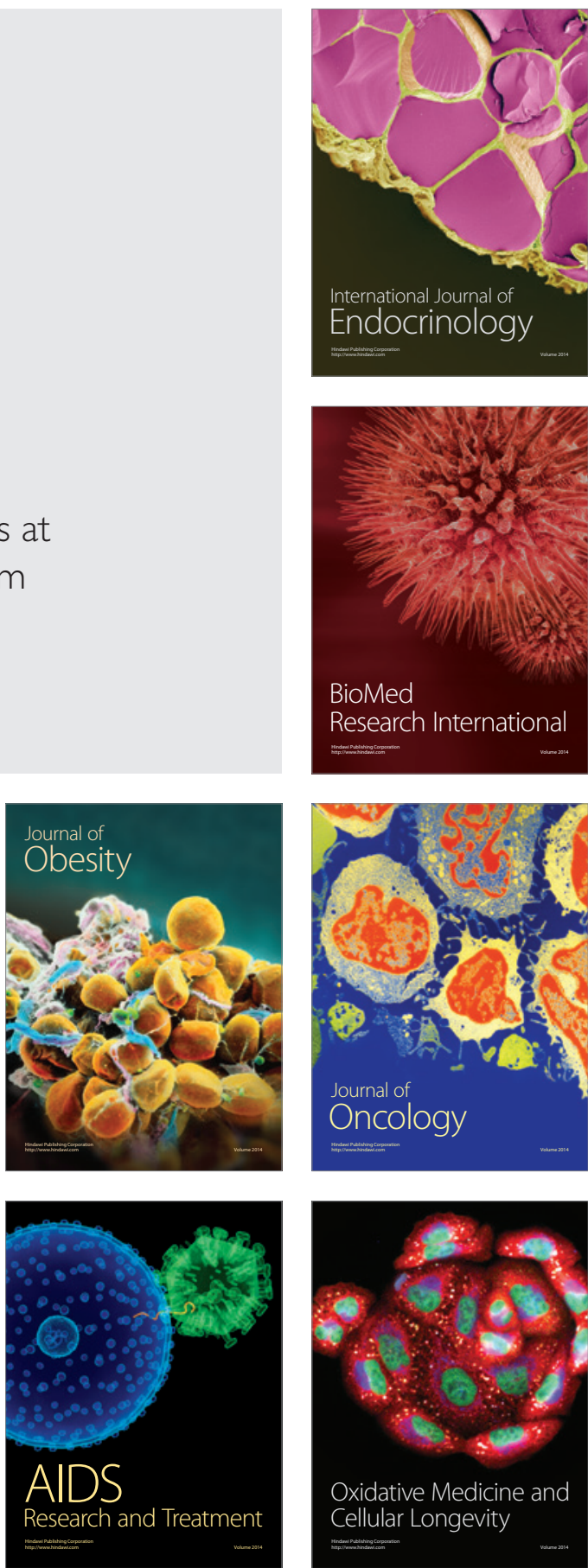